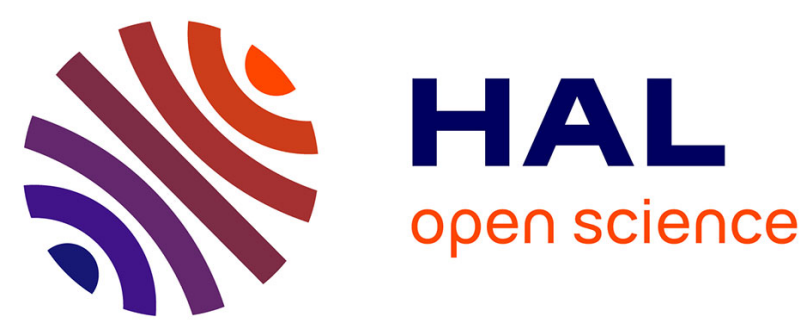

\title{
Cell adhesion regulates CDC25A expression and proliferation in acute myeloid leukemia.
}

Anne Fernandez-Vidal, Loic Ysebaert, Christine Didier, Rémy Bétous,

Fabienne de Toni, Naïs Prade-Houdellier, Cécile Demur, Marie-Odile

Contour-Galcéra, Grégoire P Prévost, Bernard Ducommun, et al.

\section{To cite this version:}

Anne Fernandez-Vidal, Loic Ysebaert, Christine Didier, Rémy Bétous, Fabienne de Toni, et al.. Cell adhesion regulates CDC25A expression and proliferation in acute myeloid leukemia.. Cancer Research, 2006, 66 (14), pp.7128-35. 10.1158/0008-5472.CAN-05-2552 . hal-00317379

\section{HAL Id: hal-00317379 \\ https://hal.science/hal-00317379}

Submitted on 26 Sep 2017

HAL is a multi-disciplinary open access archive for the deposit and dissemination of scientific research documents, whether they are published or not. The documents may come from teaching and research institutions in France or abroad, or from public or private research centers.
L'archive ouverte pluridisciplinaire HAL, est destinée au dépôt et à la diffusion de documents scientifiques de niveau recherche, publiés ou non, émanant des établissements d'enseignement et de recherche français ou étrangers, des laboratoires publics ou privés. 


\title{
Cell Adhesion Regulates CDC25A Expression and Proliferation in Acute Myeloid Leukemia
}

\author{
Anne Fernandez-Vidal, ${ }^{1}$ Loïc Ysebaert, ${ }^{1}$ Christine Didier, ${ }^{3}$ Remy Betous, ${ }^{1}$ Fabienne De Toni, ${ }^{1}$ \\ Naïs Prade-Houdellier, ${ }^{2}$ Cécile Demur, ${ }^{2}$ Marie-Odile Contour-Galcéra, ${ }^{4}$ Grégoire P. Prévost, ${ }^{4}$ \\ Bernard Ducommun, ${ }^{3}$ Bernard Payrastre, ${ }^{1}$ Claire Racaud-Sultan, ${ }^{1}$ and Stéphane Manenti ${ }^{1}$
}

${ }^{1}$ Centre de Physiopathologie Toulouse-Purpan, Institut National de la Sante et de la Recherche Medicale U563-IFR30, Département

“Oncogenèse et Signalisation dans les cellules hématopoḯtiques," and '2ervice d'Hématologie Biologique, CHU Purpan;

${ }^{3}$ Laboratoire de Biologie cellulaire et Moléculaire du Contrôle de la Prolifération, Centre National de la

Recherche Scientifique UMR 5088-IFR109, Université Paul Sabatier, Toulouse, France; and ${ }^{4}$ IPSEN,

Institut Henri Beaufour, Les Ulis, France

\begin{abstract}
The effects of cell adhesion on leukemia cell proliferation remain poorly documented and somehow controversial. In this work, we investigated the effect of adhesion to fibronectin on the proliferation of acute myeloid leukemia (AML) cell lines (U937 and KG1a) and $\mathrm{CD34}^{+}$normal or leukemic primary cells. We observed an increased rate of proliferation of AML cells when adhered to fibronectin, concomitant with accelerated S-phase entry and accumulation of CDC25A. Conversely, normal $\mathrm{CD34}^{+}$cell proliferation was decreased by adhesion to fibronectin with a concomitant drop in CDC25A expression. Importantly, we showed that both small interfering RNA (siRNA)-mediated CDC25A down-regulation and a recently developed CDC25 pharmacologic inhibitor impaired this adhesion-dependent proliferation, establishing a functional link between CDC25A accumulation and adhesion-dependent proliferation in leukemic cells. CDC25A accumulation was found only slightly dependent on transcriptional regulation and essentially due to modifications of the proteasomal degradation of the protein as shown using proteasome inhibitors and reverse transcription-PCR. Interestingly, CDC25A regulation was Chk1 dependent in these cells as suggested by siRNA-mediated down-regulation of this protein. Finally, we identified activation of the phosphatidylinositol 3-kinase/ Akt pathway as an adhesion-dependent regulation mechanism of CDC25A protein expression. Altogether, our data show that in leukemic cells adhesion to fibronectin increases CDC25A expression through proteasome- and Chk1-dependent mechanisms, resulting in enhanced proliferation. They also suggest that these adhesion-dependent proliferation properties of hematopoietic cells may be modified during leukemogenesis. (Cancer Res 2006; 66(14): 7128-35)
\end{abstract}

\section{Introduction}

Eukaryotic cells need interactions with their microenvironment to regulate their proliferation. Adhesion to the extracellular matrix (ECM) has been extensively described as a key positive regulator of cell proliferation in various cell types (1), although in some cases

Requests for reprints: Stéphane Manenti, Centre de Physiopathologie ToulousePurpan, Institut National de la Sante et de la Recherche Medicale U563-IFR30, Département "Oncogenèse et Signalisation dans les cellules hématopoïétiques," CHU Purpan, BP3028, 31024 Toulouse Cedex 3, France. Phone: 33-562-74-45-24; Fax: 33-562-

74-45-58; E-mail: stephane.manenti@toulouse.inserm.fr.

(C)2006 American Association for Cancer Research.

doi:10.1158/0008-5472.CAN-05-2552 interaction with the ECM may inhibit cell proliferation $(2,3)$. Various cell signaling pathways have been involved in this regulation (4). In response to these signal transduction pathways, cyclin D1, p21 ${ }^{\mathrm{Cip} 1}$, and $\mathrm{p} 27^{\mathrm{Kip} 1}$ appear as the main $\mathrm{G}_{1}$ cell cycle regulators downstream of cell adhesion and can be considered as molecular sensors of extracellular stimuli $(5,6)$. Both transcriptional and post-translational regulations of these proteins have been described in this context.

The microenvironment of hematopoietic stem cells and progenitors plays a crucial role in the normal and leukemic hematopoiesis in the bone marrow. Stimulation by various cytokines and direct interaction with the ECM and with neighbor cells determine differentiation and proliferation cues at the progenitor cells levels (7). For instance, $\alpha_{4}$ integrins regulate the proliferation/differentiation balance of hematopoietic progenitors (8). However, the functional and molecular effect of integrin-dependent cell adhesion to ECM on proliferation has not been extensively investigated in these cells. In normal human immature $\mathrm{CD} 34^{+}$cells, adhesion to fibronectin, a major component of the ECM in the bone marrow, inhibits cell proliferation $(9,10)$. Up-regulation of the cyclindependent kinase (CDK) inhibitor $\mathrm{p} 27^{\mathrm{Kip} 1}$ was correlated with this inhibition. By contrast, this is apparently not the case in more mature $\left(\mathrm{CD}^{-} 4^{-}\right)$cells adhered to fibronectin (11). In acute myeloid leukemia (AML) cells, the role of the adhesion to the ECM on cell proliferation remains unclear, although in one study the adhesion to fibronectin of AML cells from some patients was found to act in synergy with stem cell factor (SCF) to induce proliferation (12).

CDC25A is a dual-specificity phosphatase mostly involved in the $\mathrm{G}_{1}-\mathrm{S}$ transition of the cell cycle, although its implication in mitosis has also been recently described (13). Dephosphorylation of $\mathrm{Thr}^{14}$ and $\mathrm{Tyr}^{15}$ residues on $\mathrm{CDK} 2$ by CDC25A leads to the activation of the CDK2/cyclin E complex and participates to the $\mathrm{G}_{1}$-S-phase transition. To this respect, CDC25A can be considered as a key regulator of the $G_{1}$ phase and as a potential sensor for extracellular stimuli. Still, major regulatory factors of this protein in response to mitogenic extracellular stimuli have not been extensively documented. E2F-dependent transcriptional regulation of CDC25A was reported and found necessary for efficient E2F-dependent S-phase entry (14). In an other study, this transcriptional regulation by E2F was described as a serum-dependent mechanism (15). Transcriptional regulation of CDC25A by c-myc was also reported, although the physiologic context of this process remains unclear $(16,17)$. In addition to transcriptional mechanisms, the CDC25A protein can be subjected to proteolytic processing by the proteasome. Double-strand breaks induced on DNA by genotoxic compounds or by radiations lead to the checkpoint kinase Chk1 activation and 
subsequent CDC25A phosphorylation, triggering its degradation by the proteasome through a SCF $\beta$-TrCP-dependent mechanism (for a review, see ref. 18). Although this process was essentially described in response to genotoxic stress, recent studies suggest that this ubiquitin-proteasome system could also be involved in stress-independent CDC25A regulation during normal cell cycle (19). The question of how CDC25A levels are regulated seems of outstanding interest in cancer because overexpression of the protein has been described in various cancer cell types. In these studies, post-translational mechanisms of regulation have been found involved in the stabilization of the protein (20).

In this work, we investigated how cell adhesion to fibronectin influences the proliferation of AML cells, and we established the importance of CDC25A regulation in this context. In these malignant cells, adhesion to fibronectin up-regulates CDC25A expression through regulation of its degradation, leading to increased proliferation.

\section{Materials and Methods}

Cytokines, antibodies, and pharmacologic inhibitors. The phosphatidylinositol 3-kinase (PI3K) inhibitors LY294002 and wortmannin and the mammalian target of rapamycin (mTOR) inhibitor rapamycin were purchased from Sigma (St. Louis, MO). The p38 mitogen-activated protein kinase (MAPK) inhibitor SB203580 and the proteasome inhibitors MG132 and lactacystin were purchased from Calbiochem (La Jolla, CA). UCN-01 was provided by the National Cancer Institute (Rockville, MD). SCF, interleukin (IL)-3, Flt3 ligand (FL), and granulocyte-macrophage colony-stimulating factor (GM-CSF) were from R\&D Systems (Minneapolis, MN). Antibodies used were monoclonal anti-CDC25A, monoclonal anti-cyclin A, monoclonal anti-Chk1, polyclonal anti-CDK2, and polyclonal anti-Akt1/Akt2 (Santa Cruz Biotechnology, Santa Cruz, CA). Polyclonal anti-phospho-Ser ${ }^{473}$-Akt, anti-phospho-p38, and anti-phospho-CDC2 were from Cell Signaling (Beverly, MA). Actin monoclonal antibody $(1: 10,000)$ was from Sigma.

Cell lines and culture conditions. Leukemic immature KGla and more mature U937 and HL-60 cell lines were purchased from the German Collection of Microorganisms and Cell Cultures (Braunschweig, Germany) and grown in Iscove's modified Dulbecco's medium (IMDM) plus 20\% FCS and RPMI plus $10 \% \mathrm{FCS}$, respectively, in the presence of $100 \mathrm{units} / \mathrm{mL}$ penicillin/ $100 \mu \mathrm{g} / \mathrm{mL}$ streptomycin at $37^{\circ} \mathrm{C}$ and $5 \% \mathrm{CO}_{2}$. Jurkat cells were purchased from the German Collection of Microorganisms and Cell Cultures and grown in RPMI plus $10 \%$ FCS. Normal bone marrow $\mathrm{CD}^{+} 4^{+}$hematopoietic cells were harvested from healthy volunteers upon informed consent after positive selection of CD34-expressing cells by the means of immunomagnetic separation column (Miltenyi Biotech, Bergisch Gladbach, Germany). The purity of $\mathrm{CD}_{3} 4^{+}$cells reached $85 \%$ to $98 \%$ according to flow cytometry analysis. Leukemic blasts from patients with newly diagnosed AML and referred to the Toulouse Purpan University Hospital were obtained upon informed consent from bone marrow aspirates by Ficoll-Hypaque density gradient centrifugation. Frozen cells were thawed in EGM2 medium (ref. 21; Cambrex, Walkersville, MD) supplemented with SCF $(100 \mathrm{ng} / \mathrm{mL})$, IL-3 $(5 \mathrm{ng} / \mathrm{mL})$, FL $(100 \mathrm{ng} / \mathrm{mL})$, and GM-CSF $(10 \mathrm{ng} / \mathrm{mL})$. Cells were left at $1 \times 10^{6} / \mathrm{mL}$ for 24 hours in these conditions during which moderate cell death occurred (10-40\% of the cells depending of the patient). At that time, cells were processed for the proliferation experiments described in Results. Apoptotic cells were detected with the Annexin V-FITC detection kit from BD PharMingen (San Diego, CA) used as recommended by the manufacturer.

Fibronectin matrix preparation and 3-(4,5-dimethylthiazol-2-yl)2,5-diphenyltetrazolium bromide proliferation assay. Six-well culture dishes were coated overnight at $4^{\circ} \mathrm{C}$ with $40 \mu \mathrm{g} / \mathrm{mL}$ human fibronectin (Roche Molecular Biochemicals, Mannheim, Germany) in a final volume of $1 \mathrm{~mL}$ in PBS. Each well was rinsed twice with PBS before seeding the cells at relevant concentration (usually $0.5 \times 10^{6} / \mathrm{mL}$ for proliferation conditions). Quantification of living cells was quantified by 3-(4,5-dimethylthiazol-2-yl)2,5-diphenyltetrazolium bromide (MTT) assay (Sigma).
Differentiation of $\mathrm{CD}^{+} 4^{+}$normal progenitors in vitro. $\mathrm{CD}^{+} 4^{+}$cells were cultured and differentiated in 12-well plates in IMDM plus $10 \%$ FCS supplemented with SCF (100 ng/mL), IL-3 (5 ng/mL), FL (100 ng/mL), and GM-CSF $(10 \mathrm{ng} / \mathrm{mL})$ for myelomonocytic differentiation. Cells were initially seeded at $400,000 / \mathrm{mL}$ and diluted with fresh medium and cytokines every 2 days. Expression of the differentiation markers (CD34, CD38, CD33, and CD11b) was assessed by flow cytometry (200,000 cells used). For each time point, 300,000 cells were used for Western blot analysis.

Transfection of small interfering RNA. Transfection experiments were done using the Amaxa nucleofection technology protocols (Amaxa, Cologne, Germany). U937 cells were grown until subconfluence $\left(1 \times 10^{6} / \mathrm{mL}\right)$ and $6 \times 10^{6}$ cells were resuspended in $100 \mu \mathrm{L}$ Amaxa solution V. Eight picomoles of Chk1 small interfering RNA (siRNA; Qiagen, Valencia, CA), CDC25A SMARTpool, Aktl siRNA, or scramble siRNA (Dharmacon, Lafayette, CO) were added and cells were immediately transfected using the Amaxa nucleofector device (program V-01 for U937 and C-16 for Jurkat cells). For Akt, a second transfection was done in the same conditions 24 hours after the first one to improve the siRNA efficiency. Cells were subsequently resuspended in normal medium culture at $10^{6} / \mathrm{mL}$ and processed after 8 (U937) or 24 (Jurkat) hours for relevant experiments. Adhesion experiments were usually done at $0.5 \times 10^{6} \mathrm{cells} / \mathrm{mL}$ in the presence of $1 \%$ FCS.

Western blot analysis and immunoprecipitation. Cells $\left(2 \times 10^{6}\right)$ were usually reduced in $75 \mu \mathrm{L}$ Laemmli sample buffer, sonicated for 6 seconds, and boiled for 3 minutes. Proteins were then resolved on SDS-PAGE and transferred to nitrocellulose membrane (Hybond-C Super, Amersham Pharmacia Biotech, Piscataway, NJ). Saturation of the membrane was done for 1 hour in TBS with Tween $0.05 \%$ (TBS-T) containing $5 \%$ nonfat milk. Membranes were blotted with proper antibodies overnight at $4^{\circ} \mathrm{C}$ or for 1 hour at room temperature, washed thrice with TBS-T, and incubated for 30 minutes with horseradish peroxidase-coupled secondary antibody (Cell Signaling). After three additional washes, detection was achieved with Pierce Supersignal chemiluminescent substrate (Pierce, Rockford, IL). Antibodies were used at the following dilutions: anti-CDC25A (1:200), anti-cyclin A (1:1,000), anti-Chk1 (1:1,000), anti-Akt1/Akt2 (1:1,000), antiCDK2 (1:500), anti-phospho-Ser ${ }^{473}$-Akt (1:1,000), anti-phospho-Cdc2 (1:500), anti-phospho-p38 (1:500), and anti-actin (1:10,000). Western blot quantifications were done with the Gene Tools software from SynGene (Cambridge, United Kingdom). For CDK2 immunoprecipitation experiments, $5 \times 10^{6}$ cells were lysed in $1 \mathrm{~mL}$ immunoprecipitation buffer [50 mmol/L Tris (pH 8), $150 \mathrm{mmol} / \mathrm{L} \mathrm{NaCl}, 3 \mathrm{mmol} / \mathrm{L}$ EGTA, $1 \% \mathrm{NP} 40,50 \mathrm{mmol} / \mathrm{L} \mathrm{NaF}$, $1 \mathrm{mmol} / \mathrm{L}$ sodium orthovanadate, $10 \mathrm{mmol} / \mathrm{L} \beta$-glycerophosphate, protease inhibitors] and kept for 20 minutes on ice. After centrifugation at 14,000 rpm for 10 minutes at $4^{\circ} \mathrm{C}$, the supernatant was incubated with $5 \mu \mathrm{g}$ of the anti-CDK2 antibody and $40 \mu \mathrm{L}$ protein A-Sepharose overnight at $4^{\circ} \mathrm{C}$. Following two washes with the immunoprecipitation buffer, the immunoprecipitated material was resuspended in $60 \mu \mathrm{L}$ Laemmli sample buffer, boiled for 3 minutes, and analyzed by Western blot with the anti-Tyr ${ }^{15}$ phosphorylated CDC2 antibody (1:1,000), which also recognizes the $\mathrm{Tyr}^{15}$ phosphorylated form of CDK2.

Real-time PCR. Total RNA was extracted by guanidine isothiocyanatephenol-chloroform extraction ready-to-use TRIzol reagent (Invitrogen, Carlsbad, CA) according to the manufacturer. Extraction was done from $1 \times 10^{7}$ cells in $1 \mathrm{~mL}$ TRIzol solution. cDNA was generated from $5 \mu \mathrm{g}$ RNA, with the SuperScript reverse transcriptase from the SuperScript III FirstStrand Synthesis System for Reverse Transcription-PCR (RT-PCR; Invitrogen) following the manufacturer's suggestions. The PCR mixture containing $5 \mu \mathrm{L}$ cDNA reactant and $0.5 \mu \mathrm{mol} / \mathrm{L}$ of each primer in a final volume of $25 \mu \mathrm{L}$ prepared using the quantitative PCR Mastermix Plus SYBR Green I (Eurogentec, Seraing, Belgium). In negative controls, the reverse transcriptase was replaced by $\mathrm{H}_{2} \mathrm{O}$. The primer sequences were as follows: CDC25A 5'-ACCGTCACTATGGACCAGC-3' (sense; $0.5 \mu \mathrm{mol} / \mathrm{L}$ ) and $5^{\prime}$-TTCAGAGCTGGACTACATCC-3' (antisense; $0.5 \mu \mathrm{mol} / \mathrm{L}$ ) and glyceraldehyde-3phosphate dehydrogenase (GAPDH) 5'-TGCACCACCAACTGCTTAGC-3' (sense) and 5'-GGCATGGACTGTGGTCATGAG-3' (antisense; $0.3 \mu \mathrm{mol} / \mathrm{L}$ ). To confirm product purity, melting curves were checked and in some cases complemented by $1 \%$ agarose gel electrophoresis. 
Bromodeoxyuridine incorporation assay. The $S$ phase of the cell cycle was assayed by measuring the incorporation of bromodeoxyuridine (BrdUrd). Cells were pulse labeled with $10 \mu \mathrm{mol} / \mathrm{L}$ BrdUrd for 30 minutes, washed with PBS, and fixed in cold $70 \%$ ethanol for 20 minutes. BrdUrd detection was done by use of the BrdUrd staining kit from BD PharMingen (FITC-conjugated BrdUrd antibody set). The cells were then analyzed by flow cytometry with a FACScan cytometer (Becton Dickinson, San Diego, CA), and the percentage of cells synthesizing DNA was determined.

\section{Results}

Adhesion to fibronectin increases AML cell proliferation. To establish the effect of integrin-dependent adhesion on AML cell proliferation, U937 and KGla cell lines were grown in suboptimal (1\% FCS) conditions and maintained in suspension or adhered on a fibronectin matrix. Cells were counted daily by using a MTT colorimetric method. The results of these experiments show that after 3 days cell adhesion to fibronectin significantly increased the

A
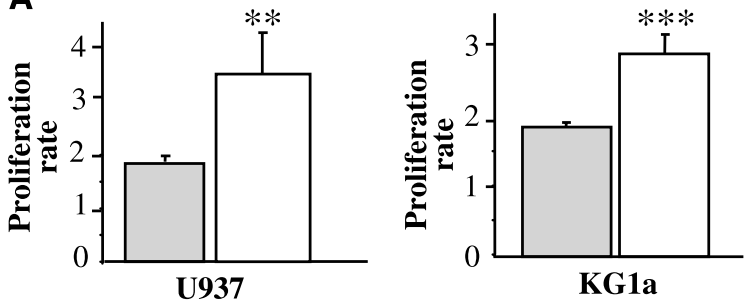

Sp Adh

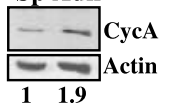

Sp Adh

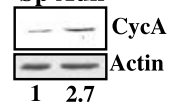

B
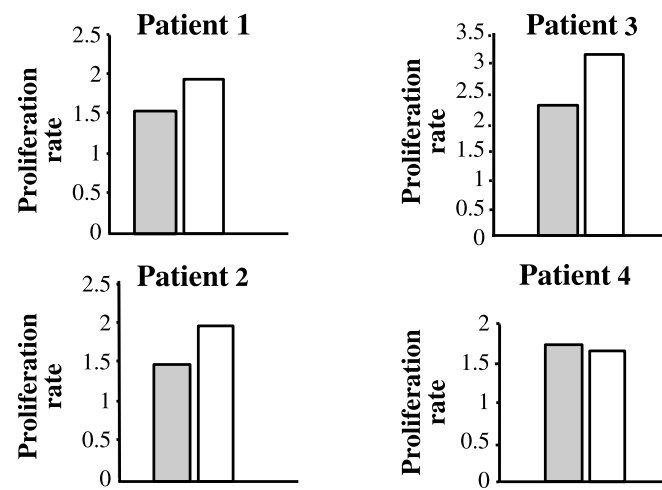

Patient 4

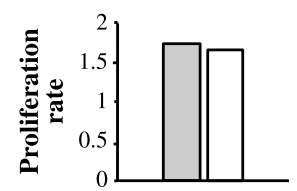

Figure 1. Adhesion to fibronectin increases the proliferation rate of AML cells. $A$, U937 and KG1a cell lines were grown in the presence of $1 \%$ and $10 \%$ FCS, respectively, in normal suspension conditions ( $S p$; gray columns) or in the presence of a fibronectin matrix ( $A d h$; white columns). Viable cells were counted daily by the MTT method. The day $3 /$ day 0 ratio was calculated from the corresponding absorbance values. Columns, mean of four independent experiments done in triplicate; bars, SD. ${ }^{\star \star}, P<0.01 ;{ }^{\star \star \star}, P<0.001$, compared with suspension conditions. U937 and KG1a cells were left in suspension or adherent for 14 hours, and cyclin A expression was analyzed by Western blot after that time. Densitometric quantification of the Western blot signal was done, and the ratio between the CDC25A and the corresponding actin value was indicated for each fraction. $B, A M L$ blasts from patients were processed as described in Materials and Methods and used for proliferation experiment. The number of cells was counted each 48 hours, and the proliferation rate was calculated as in $(A)$ and compared in the suspension (gray columns) and adhesion (white columns) culture conditions. Except for patient 1, in which cells underwent proliferation at day 1, proliferation between days 3 and 5 is shown for the other three patients. proliferation rate of both U937 and KGla leukemic cells (Fig. 1A). Annexin V-FITC apoptosis detection experiments were done as a control, indicating no significant difference of apoptosis between adherent and nonadherent cells (data not shown). BrdUrd incorporation experiments indicated an increase of $21.4 \pm 2 \%$ (two experiments) and $16.5 \pm 1.8 \%$ (three experiments) in S-phase entry of U937 and KGla cells, respectively, on adhesion to fibronectin. To confirm this higher proliferation rate at the molecular level, we checked the expression of cyclin A, which is considered as a biochemical marker of the S-phase entry during the cell cycle. As can be seen in Fig. $1 A$ (insets), cyclin A expression was significantly increased (1.9- and 2.7-fold in U937 and KGla, respectively) when cells were adhered to fibronectin for 14 hours, consistent with the positive effect of cell adhesion on proliferation.

To confirm these data, we did similar experiments with AML blasts from patients. Four patients with an immature phenotype $\left(\mathrm{CD} 34^{+}, \mathrm{M} 1\right)$ were used for this experiment (see results in Fig. 2 for justification). Cells were thawed in EGM2 medium in the presence of SCF, GM-CSF, IL-3, and FL, placed on a fibronectin matrix or left in suspension, and counted every 48 hours. As can be seen in Fig. $1 B$, AML blasts from three of these patients showed increased proliferation when adhered to fibronectin, confirming the data obtained with the established cell lines. Altogether, these data show that cell adhesion to fibronectin increases the proliferation of AML cells.

Adhesion to fibronectin differently affects the proliferation of normal hematopoietic cells depending on their maturation state. To establish whether the above-mentioned adhesiondependent proliferation increase was specific of AML cells, we checked the effect of cell adhesion on the proliferation of normal hematopoietic progenitors from healthy donors. For this purpose, $\mathrm{CD}_{3}{ }^{+}$normal hematopoietic cells were grown for 2 weeks in cytokine-supplemented medium conditions, allowing their differentiation along with the myelomonocytic pathway. This maturation process was conducted either in suspension or in adhesion conditions on fibronectin. The proliferation rate and the differentiation state were monitored every 2 days by direct counting of the living cells and by fluorescence-activated cell sorting (FACS) analysis, respectively. The results of these experiments indicate that proliferation of immature $\mathrm{CD} 34^{+}$cells is inhibited on binding to the fibronectin matrix (Fig. 2, top). By contrast, adhesion to fibronectin of more mature $\mathrm{CD} 34^{-}$cells stimulated their proliferation (Fig. 2, bottom). These data suggest that the effect of integrin-dependent adhesion on cell proliferation depends on the differentiation stage of normal hematopoietic cells. They also point to an opposite behavior of normal and leukemic (see results with KGla cell line and blasts from patients in Fig. 1) immature CD34 ${ }^{+}$ cells in terms of adhesion-dependent cell proliferation.

Adhesion to fibronectin up-regulates CDC25A protein level in AML cells. Cyclin D1, p27 $7^{\mathrm{Kip} 1}$, and $\mathrm{p} 21^{\mathrm{Cip} 1}$ are key cell cycle regulators involved in extracellular signals integration during the $\mathrm{G}_{1}$ phase of the cell cycle. To investigate the mechanisms of this anchorage-dependent proliferation increase, we checked the expression of these proteins by Western blot. In the experimental conditions used for proliferation studies, we did not observe any significant modification of the cellular expression levels for these proteins (data not shown). By contrast, we observed a dramatic increase of the dual-specificity phosphatase CDC25A, an other important player of the $\mathrm{G}_{1}-\mathrm{S}$ transition (Fig. $3 A$ ). This process was observed in the two previously described AML cell lines U937 and KGla but also in the myeloblastic CD34 ${ }^{-}$HL-60 cell line. These 


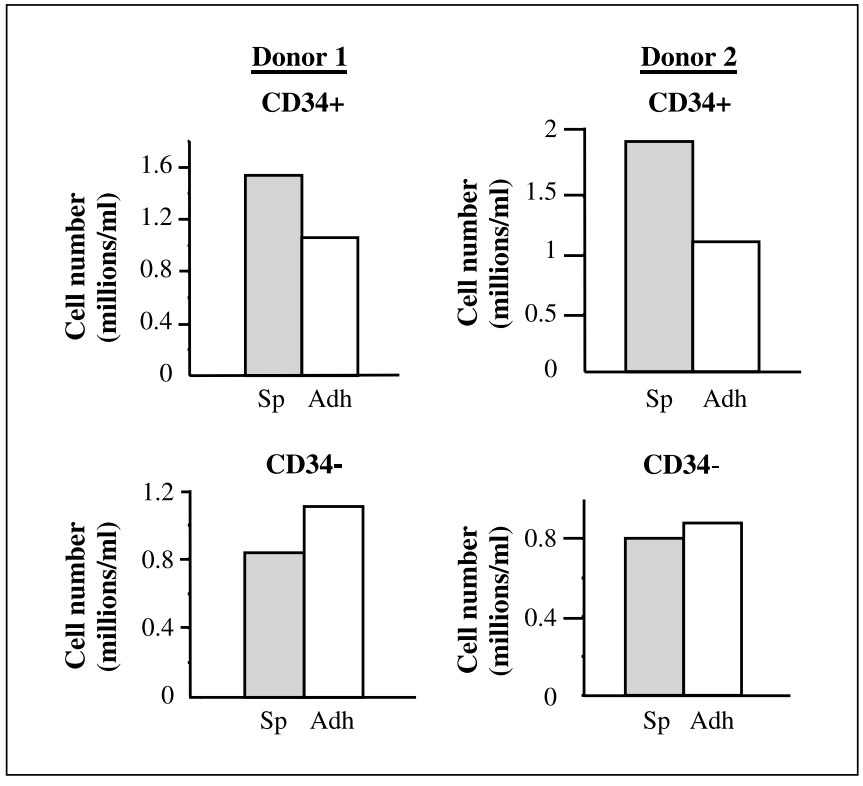

Figure 2. Adhesion to fibronectin inhibits or stimulates the proliferation of normal hematopoietic cells depending on their maturation state. Adherent (white columns) or suspension (gray columns) normal hematopoietic CD34 ${ }^{+}$ cells were differentiated in vitro as described in Materials and Methods. The proliferation was compared at two different stages $\left(\mathrm{CD} 34^{+}\right.$and $\left.\mathrm{CD} 34^{-}\right)$of the differentiation process. Results from two independent donors (donors 1 and 2). The cell number 3 days after seeding was compared for the adhesion and suspension conditions. Results are expressed in cell number $/ \mathrm{mL}$. For donor 1 , the proliferation experiments were done between days 3 and 5 and days 10 and 12 for CD $34^{+}$and CD34- cells, respectively. For donor 2 , proliferations were done between days 1 and 3 and days 8 and 10, respectively. This corresponded to similar stages of differentiation for the two donors as judged by FACS analysis of cell surface markers (data not shown).

results suggest for the first time the existence of an adhesiondependent regulation of CDC25A expression.

We then tested the effect of cell adhesion on CDC25A expression in blasts from patients with immature $\mathrm{CD} 34^{+}$and $\mathrm{M} 1$ phenotype. As can be seen in Fig. $3 B$, blasts from patients 2 and 3 showed increased CDC25A expression when adhered to fibronectin, in good correlation with the proliferation experiments (see Fig. 1). Blasts from a third patient (patient 5), not tested for proliferation, also showed CDC25A expression increase in adhesive conditions. CDC25A level was found unchanged in patients 1 and 4 (data not shown). These data obtained with blasts from patients confirm that CDC25A protein is up-regulated in AML cells following adhesion to fibronectin.

We then examined this regulation process in normal hematopoietic cells. As shown in Fig. 3C, CDC25A expression was high in $\mathrm{CD}_{3} 4^{+}$immature cells and dramatically reduced on adhesion to fibronectin. By contrast, the expression level of this protein was lower in more differentiated $\mathrm{CD}_{4} 4^{-}$cells and significantly increased by the adhesion to fibronectin. These data suggest that (a) CDC25A protein level is regulated along the hematopoietic differentiation process and that cell interaction with the ECM may differently affect this expression and $(b)$ up-regulation of CDC25A on adhesion is not maturation restricted in AML as opposed to their normal counterparts.

We finally tested the adhesion-dependent regulation of CDC25A in other hematologic malignancies. Jurkat cells, the Karpas large cell anaplastic lymphoma cell line, the ES mantle lymphoma cell line, and the follicular lymphoma RL and Karpas 422 cell lines were tested to this respect. As shown in Fig. $3 D$, the effect of cell adhesion on CDC25A protein level is clearly different from one cell type to the other. On adhesion to fibronectin, CDC25A increased in Jurkat and RL cells, decreased in Karpas 422 cells, and remained low and apparently unchanged in Karpas and ES cells. These data indicate that adhesion-dependent regulation of CDC25A is not restricted to AML cells and they confirm that this regulation may lead to opposite effects on CDC25A level.

CDC25A accumulation is involved in adhesion-dependent proliferation. We then addressed the question of the functional relationship between adhesion-dependent CDC25A expression and increased cell proliferation. To this purpose, we down-regulated CDC25A expression in U937 cells by a siRNA strategy and checked the adhesion-dependent cyclin A expression by Western blot. As observed in Fig. $4 A$, a moderate siRNA-induced down-regulation of CDC25A significantly decreased the cyclin A protein level in adherent cells. These data suggest that modifications of CDC25A

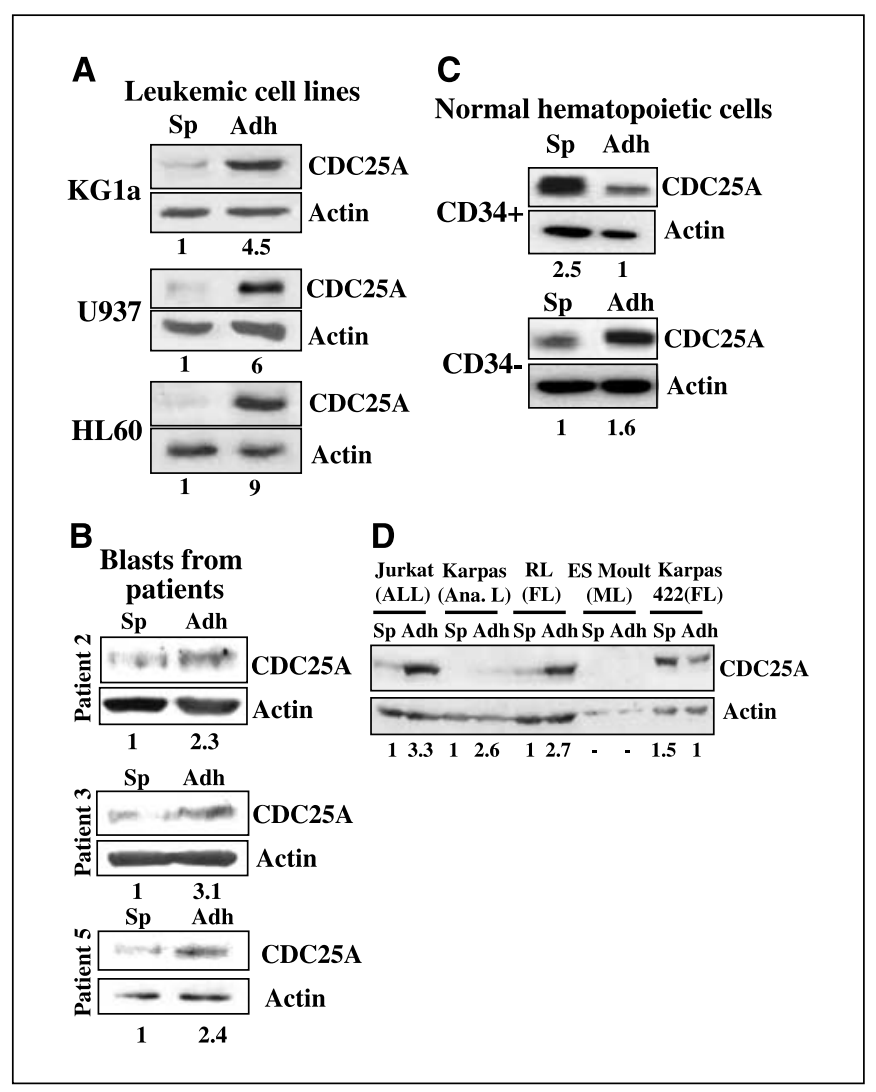

Figure 3. CDC25A phosphatase expression is regulated by cell adhesion to fibronectin. $A, \mathrm{AML}$ cell lines were either adhered to fibronectin for 14 hours or left in suspension for the same time. CDC25A level was then visualized by Western blot. Membranes were then stripped and blotted again with an antibody against actin. $B$, blasts from patients were cultured on fibronectin or left in suspension for 14 hours and then processed for Western blotting analysis. In all cases, densitometric quantification of the Western blot signal was done, and the ratio between the CDC25A and the corresponding actin value was indicated for each fraction. $C$, normal hematopoietic $C D 34^{+}$cells were subjected to a differentiation process in vitro as described before. At day 0 (CD34 ${ }^{+}$status) and day 7 (CD34 ${ }^{-}$status), cells were collected and adhered to fibronectin matrix or left in suspension for 14 hours. CDC25A expression was then detected by Western blot analysis on the corresponding cellular extracts. These results were reproduced in at least three independent experiments for each cell line and twice for the primary cells. $D$, five cell lines, from various hematologic malignancies, were tested by Western blotting for CDC25A expression in suspension and adhesion conditions. Representative of two independent experiments. $A L L$, acute lymphoblastic leukemia; $M L$, mantle lymphoma; $F L$, follicular lymphoma; Ana.L, anaplastic lymphoma. 
A

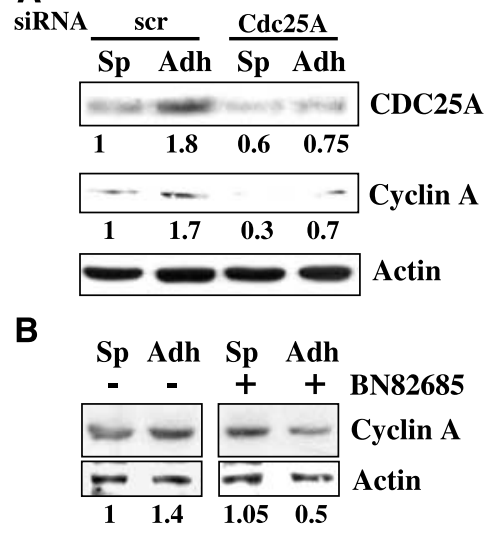

C

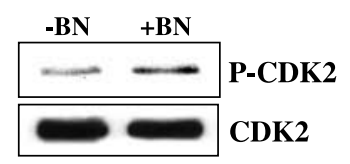

Figure 4. CDC25A regulates adhesion-dependent proliferation of AML cells. $A$, U937 cells were transfected by scramble (control; scr) or CDC25A siRNA using the Amaxa nucleofection device. After 8 hours, cells were either adhered to fibronectin or left in suspension for 14 additional hours. CDC25A and cyclin A proteins levels were then detected by Western blot in the corresponding cellular extracts. Representative of three independent experiments. B, U937 cells were adhered or left in suspension for 14 hours in the presence $(+)$ or absence $(-)$ of the CDC25 inhibitor BN82685 (100 nmol/L). Western blot analysis of the cyclin A protein was then done. These experiments were done twice. In all cases, densitometric quantification of the Western blot signal was done, and the ratio between the CDC25A and the corresponding actin value was indicated for each fraction. $C$, cells were treated with BN82685 (300 nmol/L) for 4 hours and then processed for immunoprecipitation of CDK2 as described in Materials and Methods. The inactive form of CDK2 was then detected by Western blot with an anti-phospho-Tyr ${ }^{15}$ antibody, and total immunoprecipitated CDK2 was detected with the antibody used for the immunoprecipitation.

expression drive the augmentation of proliferation triggered by cell adhesion to fibronectin.

To reinforce these observations, we made use of BN82685, a recently described CDC25 pharmacologic inhibitor (22). Because we address a question concerning the $\mathrm{G}_{1}$-S transition, we reasoned that in our experimental conditions the effect of this inhibitor should essentially concern the CDC25A phosphatase and not CDC25B or CDC25C, although these two isoforms are also inhibited in these conditions. When U937 cells were adhered to fibronectin in the presence of this inhibitor, we observed that cyclin A accumulation was impaired, in good correlation with the siRNA experiments (Fig. 4B). Altogether, these data show that CDC25A up-regulation is involved in the adhesion-dependent proliferation increase of AML cells. To confirm the effect of BN82685 on CDK2 activity, we did an immunoprecipitation of CDK2 from control and BN82685-treated cells and detected the inactive form of the kinase by Western blot with an antibody recognizing $\mathrm{Tyr}^{15}$-phosphorylated CDK2. As shown in Fig. $4 C$, BN82685 indeed increased the inhibitory phosphorylation state of CDK2 in these experiments.

Cell type-dependent variations of CDC25A mRNA on adhesion to fibronectin. To establish if transcriptional activation could control the CDC25A protein accumulation, we compared CDC25A mRNA levels in adherent and nonadherent AML cells. Quantitative RT-PCR was done for this purpose. GAPDH mRNA levels were used to normalize the measured values, and the results

were expressed in fold increase between adhesion and suspension conditions (Fig. 5). The results of these experiments indicate almost no variation of CDC25A mRNA in U937 and HL-60 cell lines (1.6 and 1.4, respectively) and a moderate increase in KGla adherent cells (4.5). These data suggest that transcriptional increase and/or mRNA stabilization are probably not involved in CDC25A protein accumulation in U937 and HL-60 cell lines, although they possibly participate to this regulation in KGla cells. In consequence, they also suggest some cell type-dependent variations of this regulation.

Involvement of Chk1 and proteasomal degradation in CDC25A turnover. We then investigated whether the previously described post-translational regulation of CDC25A was involved in the accumulation of this protein on adhesion. We focused our attention on proteasomal degradation of the protein and on the potential role of the checkpoint kinase Chkl in this process. When AML cell lines were incubated with the proteasome inhibitor MG132 for 5 hours in growing culture conditions, an accumulation of CDC25A protein was observed in each cell line (Fig. 6A). When U937 cells were transfected with Chkl siRNA, leading to major down-regulation of this protein, a significant increase of CDC25A expression was also observed (Fig. 6B). Altogether, these data suggest that phosphorylation by Chk1 and subsequent proteasomal degradation are key regulators of CDC25A protein in the absence of genotoxic stress in these cells. To confirm a role for Chkl in the adhesion-dependent regulation of CDC25A, adherent U937 and KGla cells were removed from the fibronectin matrix with EDTA in the presence of the Chk1 inhibitor UCN-01. First, removing the cells from the fibronectin matrix with EDTA induced rapid downregulation of the CDC25A protein, indicating that the adhesiondependent accumulation process was quickly reversible. As can be seen in Fig. $6 C$, the presence of UCN-01 impaired this CDC25A down-regulation, suggesting that Chk1 is actually involved in the adhesion-dependent regulation of the phosphatase. We then questioned the involvement of proteasome regulation in adhesion-dependent CDC25A increase. To this aim, the same experiment as in Fig. $6 C$ was conducted in the presence of proteasome inhibitors instead of UCN-01. The results of these experiments are presented in Fig. $6 \mathrm{D}$. The presence of proteasome inhibitors completely or partially inhibited this down-regulation depending on the cell type and on the pharmacologic inhibitor used. Altogether, these data suggest that Chk1 and the proteasome machinery are actually involved in the anchorage-dependent CDC25A regulation.

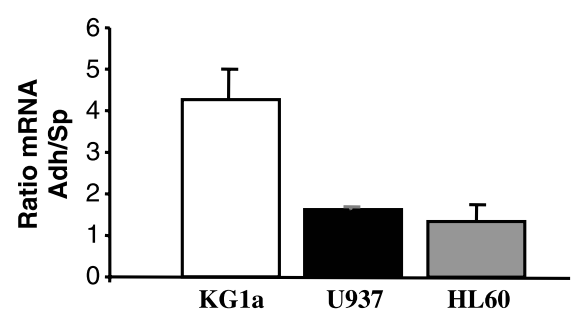

Figure 5. RT-PCR analysis of CDC25A expression in adhesion and suspension conditions. Total mRNA was prepared from adherent (14 hours) and nonadherent cells and quantitative RT-PCR was done to quantify CDC25A mRNA as described in Materials and Methods. The ratio for adherent versus suspension cells from the three cell lines was then deduced and plotted. Columns, mean of three independent experiments done in triplicate; bars, SD. 


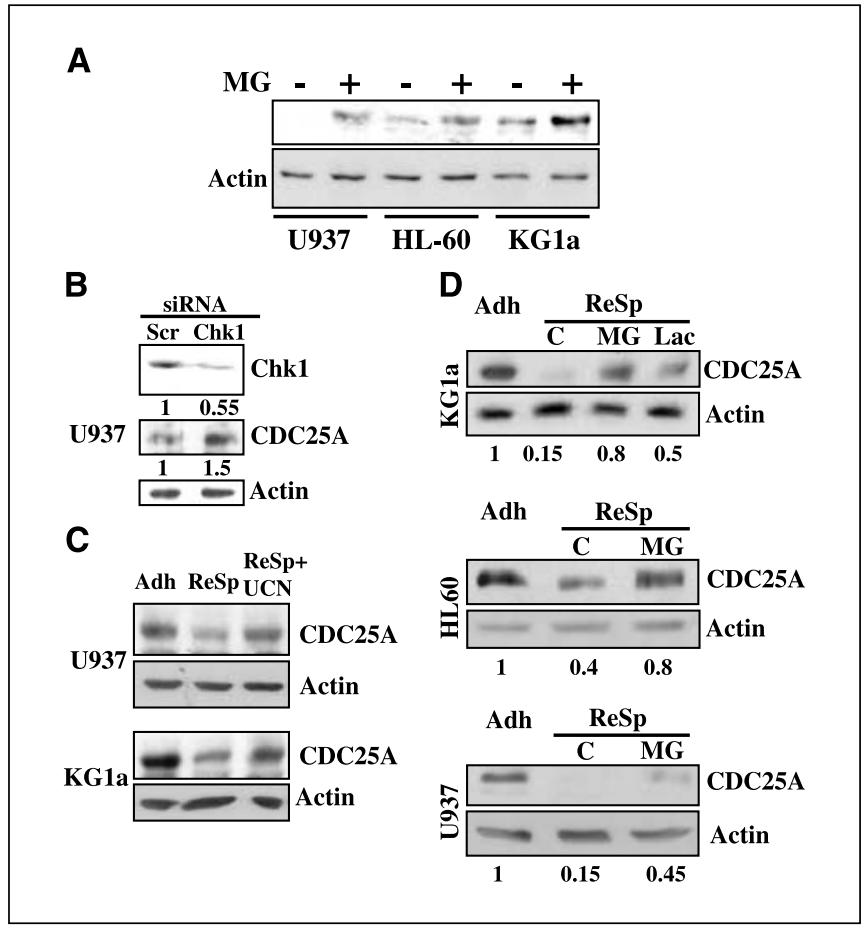

Figure 6. Proteasome- and Chk1-dependent regulation of CDC25A in AML cells. $A$, U937, $\mathrm{HL}-60$, and $\mathrm{KG1a}$ cells were incubated with the proteasome inhibitor MG132 (MG; $5 \mu \mathrm{mol} / \mathrm{L})$ for 5 hours. CDC25A level was then detected by Western blot. $B$, U937 cells were transfected with scramble or Chk1 siRNA with the Amaxa nucleofection system. After 24 hours, cell extracts were prepared and Chk1 and CDC25A expression was detected by Western blot. C, U937 and KG1a cells were adhered to fibronectin for 14 hours, removed from the fibronectin matrix with EDTA $(R e S p)$, and left in suspension conditions in the presence of UCN-01 (UCN; $100 \mathrm{nmol} / \mathrm{L})$ or with DMSO. D, as in $(C), \mathrm{U} 937$, $\mathrm{HL}-60$, and KG1a cells were adhered to fibronectin for 14 hours, removed from the fibronectin matrix with EDTA, and left in suspension conditions in the presence of the proteasome inhibitors MG132 (5 $\mu \mathrm{mol} / \mathrm{L})$ and lactacystin ( $L a c$; $5 \mu \mathrm{mol} / \mathrm{L})$ or with DMSO $(C)$. Western blot analysis of the corresponding cellular fractions was then done to follow CDC25A expression. Representative data of at least two independent experiments done from each cell line. In all cases, densitometric quantification of the Western blot signal was done, and the ratio between the CDC25A and the corresponding actin value was indicated for each fraction.

PI3K/mTOR pathway is involved in adhesion-dependent CDC25A regulation. Because the $\mathrm{p} 38$ MAPK pathway was recently involved in CDC25A protein expression in $\mathrm{T}$ lymphocytes, we investigated p38 involvement in U937 and KGla cell proliferation in suspension and adhesion conditions. The p38 inhibitor SB203580 did not affect CDC25A levels in any of the conditions or cell lines tested, suggesting that p38 MAPK is not a major regulator of CDC25A expression in AML (data not shown).

A few reports from the literature suggest that the PI3K/mTOR pathway can regulate CDC25A level in certain conditions $(23,24)$. Therefore, we tested the effect of PI3K and mTOR inhibitors on anchorage-dependent CDC25A accumulation. First, we checked the effect of cell adhesion on the activity of the PI3K pathway. For this purpose, U937 were adhered to fibronectin for different times, and the phosphorylation state of protein kinase $\mathrm{B}$ (PKB)/Akt was detected by Western blot. As shown in Fig. $7 A$, rapid and sustained activation of this pathway was observed on adhesion of U937 cells to fibronectin. Interestingly, this adhesion-dependent activation of $\mathrm{PKB} /$ Akt was also observed in AML primary cells from patients (Fig. $7 B$ ). As shown in Fig. $7 C$ and $D$, the PI3K inhibitors LY294002 and wortmannin and the mTOR-specific inhibitor rapamycin strongly impaired the adhesion-dependent CDC25A up-regulation, suggesting the participation of this pathway in this process. To further show the involvement of the PI3K pathway in CDC25A regulation, we aimed to perform siRNA-dependent Akt downregulation. Due to technical difficulties to use the U937 cells for this purpose, we used the Jurkat cell line found previously (see Fig. 3) to regulate CDC25A level through an adhesion-dependent mechanism. BrdUrd incorporation experiments done with this cell line showed increased DNA synthesis $(17.7 \pm 2.5 \%)$ on cell adhesion to fibronectin. We then verified that the PI3K inhibitors LY294002 and wortmannin impaired the CDC25A protein level in this cell type (Fig. 8A). In good agreement, siRNA-mediated downregulation of the Akt protein led to reduced expression of CDC25A in adherent cells (Fig. 8B) despite incomplete (50\%) downregulation of Akt protein in the conditions used. Altogether, these data suggest that interaction with fibronectin activates the PI3K/ Akt/mTOR pathway, which participates to CDC25A up-regulation in these conditions.

\section{Discussion}

In this work, we show that adhesion to fibronectin stimulates the proliferation of AML cell lines. This question had not been extensively addressed until now, although the proliferation state of leukemic cells in the bone marrow may be of outstanding
A

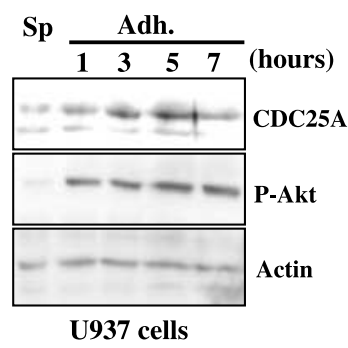

C

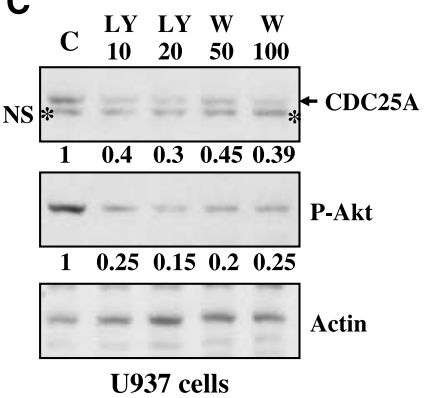

B Blasts from patients

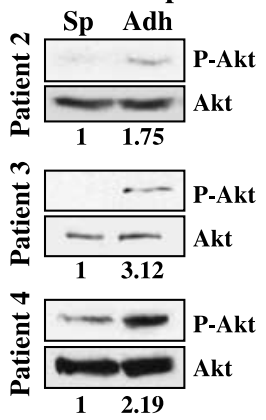

D

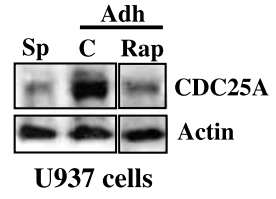

Figure 7. PI3K pathway regulates adhesion-dependent CDC25A protein accumulation. $A$, U937 cells were adhered to fibronectin for different times or left in suspension, and CDC25A expression and Akt/PKB Ser ${ }^{473}$ phosphorylation state $(P$-Akt $)$ were probed by Western blot. $B$, blasts from AML patients were left in suspension or adhered to fibronectin for 14 hours, and Akt/PKB Ser ${ }^{473}$ phosphorylation and Akt protein level were then probed by Western blot analysis. $C$, U937 cells were adhered to fibronectin for 14 hours in the absence $(C)$ or presence of the PI3K inhibitors LY294002 at $10($ LY10) or 20 (LY20) $\mu \mathrm{mol} / \mathrm{L}$ or wortmannin at 50 (W50) or $100($ W100) nmol/L. CDC25A expression and Akt/ PKB Ser ${ }^{473}$ phosphorylation state were then probed by Western blot.

Representative of four independent experiments. D, U937 cells were left in suspension conditions or adhered to fibronectin in the absence or presence of the mTOR inhibitor rapamycin (Rap; $10 \mathrm{nmol} / \mathrm{L})$. Western blot analysis of CDC25A expression was then done. Representative of two independent experiments. 


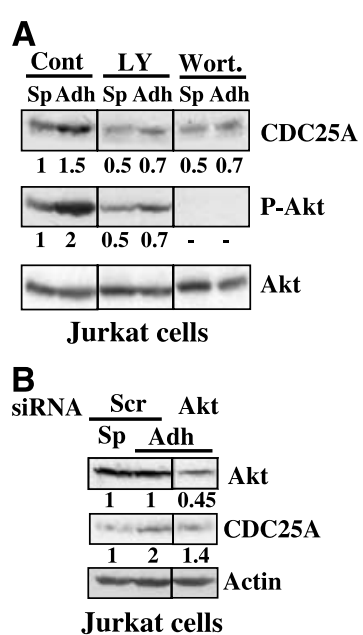

Figure 8. PKB/Akt down-regulation affects CDC25A level in Jurkat cells. $A$, Jurkat cells were left in suspension or adhered to fibronectin for 14 hours in the absence (Cont) or the presence of LY294002 ( $L Y ; 20 \mu \mathrm{mol} / \mathrm{L})$ or wortmannin (Wort.; $100 \mathrm{nmol} / \mathrm{L}$ ). CDC25A expression, Akt protein level, and Akt/PKB $\mathrm{Ser}^{473}$ phosphorylation state were then probed by Western blot. Representative of three experiments. $B$, Jurkat cells were transfected with scramble (Scr) or Akt siRNA as described in Materials and Methods and then adhered to fibronectin for 14 hours. Akt and CDC25A expression was then probed by Western blot analysis. Representative of two independent experiments.

importance for their response to therapeutic drugs and/or relapse of the disease. A synergistic effect of cell adhesion on SCF-mediated AML cell proliferation has been already reported in some cases of leukemic blasts from patients (12). Our experiments done on established cell lines and blasts from patients further reinforce these data and suggest that blast adhesion to fibronectin in the bone marrow may be a key variable amplifying the abnormal expansion of the leukemic clone. Of special interest are the results obtained with normal immature $\left(\mathrm{CD} 34^{+}\right)$cells. Adhesion onto a fibronectin matrix inhibits their proliferation as already reported by others $(9,10)$. One of the important observations is that this phenotype apparently disappears along with the myeloid differentiation process and mirrors the kinetics of CD34 antigen expression. Indeed, a more mature phenotype corresponding to CD34 ${ }^{-}$committed precursors showed accelerated proliferation on adhesion to fibronectin. Conversely, the maturation state, as assessed by French-American-British classification, does not seem anymore as a critical factor for the adhesion-dependent proliferation of leukemic cells, because both immature (CD34 $4^{+}$AML cells (KGla cell line and blasts from patients) and U937, a more mature myelomonocytic $\left(\mathrm{CD} 34^{-}\right)$cell line, showed increased anchoragedependent proliferation. The opposite effects on proliferation triggered by adhesion observed between normal and leukemic $\mathrm{CD}^{+} 4^{+}$cells suggest that modifications of the proliferative response to cell adhesion could be a critical step occurring during the leukemogenic process. Experiments are presently being done in our laboratory to answer this question.

An other important observation is that CDC25A expression was dramatically dependent on the adhesive status of the cells. To our knowledge, this is the first report of an integrin-dependent regulation of $\mathrm{CDC} 25 \mathrm{~A}$. As a general matter, there are not many reports of extracellular signal-dependent variations of CDC25A protein level in the literature. Serum- and E2F-dependent increase of CDC25A mRNA has been described, but the status of the protein was poorly documented in these studies $(14,15)$. Furthermore, our quantitative RT-PCR analysis of CDC25A mRNA does not argue for a major transcriptional regulation of the protein in our system. Our data are more in favor of post-translational regulation(s) of the protein by the proteasome as suggested by the experiments done with pharmacologic inhibitors of this machinery. However, the 4.5-fold increase of CDC25A mRNA in KGla cells on adhesion may suggest that transcriptional or mRNA stability regulations are active in certain cell types. Further experiments are needed to confirm these hypotheses. One of the important questions ensuing from our data is the cell specificity of this adhesion-dependent CDC25A regulation. The experiments done with other hematologic malignancies suggest that this regulation is not specific of AML cells. Although we did not perform proliferation experiments with these different cell lines, one may notice that increased proliferation of Jurkat cells has been described in response to cell adhesion (see ref. 25), in good agreement with the CDC25A accumulation observed in these cells (see Fig. 3) and with the increased BrdUrd incorporation that we observed on cell adhesion. Finally, although preliminary experiments from our laboratory indicate that nonhematopoietic cell lines (HeLa and HEK293) do not significantly change their CDC25A expression on cell anchorage removal, we cannot rule out the existence of this regulation in such cell types.

Our data also suggest the involvement of the PI3K pathway and of the Chk1 kinase in the adhesion-dependent regulation of CDC25A. We did not detect any adhesion-regulated modulation of Chk1 activity by Western blot with an anti-phospho-Ser ${ }^{345}$ antibody (data not shown). This does not rule out the existence of some other type(s) of regulation not detectable by this approach. Interestingly, recent publications describe the phosphorylation of Chkl by Akt and the subsequent inactivation of the kinase by cytoplasmic sequestration following monoubiquitination $(26,27)$. This hypothesis would nicely fit with our observations. Indeed, cell anchorage triggers Akt activation in our system and PI3K inhibition impairs the adhesion-dependent CDC25A up-regulation. Interestingly, a few data from the literature already ascribed a role for the PTEN/PI3K (23) and/or mTOR (24) pathways in CDC25A protein accumulation. Furthermore, the PI3K/Akt/mTOR pathway is currently deregulated in AML cells from patients (28), and we recently described the mTOR kinase as an interesting therapeutic target in these pathologies (29). Whether an integrin/PI3K/Akt/ Chk1 pathway regulates CDC25A expression in AML cells is currently under study in our laboratory.

As indicated in Results, a recent work (30) described a major role for CDC25A in the proliferation of lymphocytes. Theses authors described a direct phosphorylation of CDC25A by the p38 MAPK pathway in cytokine-starved lymphocytes and the subsequent down-regulation of the phosphatase. Although we observed some regulation of the p38 MAPK activity on cell adhesion in KGla cells, this pathway is apparently not involved in the adhesion-dependent CDC25A regulation. This discrepancy argues for different mechanisms of CDC25A regulation downstream of various mitogenic signals (growth factors, cell adhesion, etc.) and/or a cell typedependent regulation of the phosphatase.

Our data argue for the involvement of CDC25A in the adhesiondependent proliferation increase of AML cells. Whether cell adhesion to the ECM participates to the leukemogenic proliferation in vivo remains to be established, but if this was the case this would make of $\mathrm{CDC} 25 \mathrm{~A}$ an interesting therapeutic target for these 
pathologies. Of outstanding interest is the fact that the expression of this protein seems regulated during the normal hematopoietic process and permanently under the positive (mature progenitors) or negative (immature progenitors) control of integrin functions.

\section{Acknowledgments}

Received 7/20/2005; revised 4/19/2006; accepted 5/19/2006.
Grant support: Institut National de la Sante et de la Recherche Medicale, Association Laurette Fugain grant R05027BB, and Association pour la Recherche sur le Cancer grant 3638.

The costs of publication of this article were defrayed in part by the payment of page charges. This article must therefore be hereby marked advertisement in accordance with 18 U.S.C. Section 1734 solely to indicate this fact.

We thank Pierre Cavailles and Marie-Michele Duplantier for helpful expertise and discussions on real-time quantitative PCR, Martin Carroll for helpful discussions about the culture of blasts from patients, Christine Bezombes for providing us with lymphoblastic cell lines, and the Division of Cancer Treatment and Diagnosis, National Cancer Institute, for providing us with UCN-01.

\section{References}

1. Danen E, Yamada K. Fibronectin, integrins and growth control. J Cell Physiol 2001;189:1-13.

2. Meredith J, Takada Y, Fornaro M, Languino LR, Schwartz MA. Inhibition of cell cycle progression by the alternatively spliced integrin $\beta_{1 \mathrm{C}}$. Science 1995;269:1570-2.

3. Fornaro M, Steger CA, Bennett AM, Wu JJ, Languino L. Differential role of $\beta_{1 \mathrm{C}}$ and $\beta_{1 \mathrm{~A}}$ integrin cytoplasmic variants in modulating focal adhesion kinase, protein kinase B/AKT, and Ras/mitogen-activated protein kinase pathways. Mol Biol Cell 2000;11:2235-49.

4. Lee JW, Juliano R. Mitogenic signal transduction by integrin and growth factor receptor-mediated pathways. Mol Cells 2004;17:188-202.

5. Assoian R. Anchorage-dependent cell cycle progression. J Cell Biol 1997;136:1-4.

6. Assoian RK, Schwartz MA. Coordinate signaling by integrins and receptor tyrosine kinases in the regulation of $\mathrm{G}_{1}$ phase cell-cycle progression. Genes Dev 2001;11:48-53.

7. Verfaillie C. Adhesion receptors as regulator of hematopoietic process. Blood 1998;92:2609-12.

8. Arroyo AG, Yang JT, Rayburn H, Hynes RO. $\alpha_{4}$ Integrins regulate the proliferation/differentiation balance of multilineage hematopoietic progenitors in vivo. Immunity 1999;11:555-66.

9. Hurley RW, McCarthy JB, Verfaillie CN. Direct adhesion to bone marrow stroma via fibronectin receptors inhibits hematopoietic progenitor proliferation. J Clin Invest 1995;96:511-9.

10. Jiang Y, Prosper F, Verfaillie CM. Opposing effects of engagement of integrins and stimulation of cytokine receptors on cell cycle progression of normal human hematopoietic progenitors. Blood 2000;95:846-54.

11. Dylla SJ, Deyle DM, Theunissen K, Padurean AM, Verfaillie CM. Integrin engagement-induced inhibition of human myelopoiesis is mediated by proline-rich tyrosine kinase 2 gene products. Exp Hematol 2004;32: 365-74.

12. Bendall LJ, Makrynikola V, Hutchinson A, Bianchi AC Bradstock KF, Gottlieb DJ. Stem cell factor enhances the adhesion of AML cells to fibronectin and augments fibronectin-mediated anti-apoptotic and proliferative signals. Leukemia 1998;12:1375-82.

13. Mailand N, Podtelejnikov AV, Groth A, Mann M, Bartek J, Lukas J. Regulation of $\mathrm{G}_{2}-\mathrm{M}$ events by CDC25A through phosphorylation-dependent modulation of its stability. EMBO J 2002;21:5911-20.

14. Vigo E, Muller H, Prosperini E, et al. CDC25A phosphatase is a target of $\mathrm{E} 2 \mathrm{~F}$ and is required for efficient E2F-induced S phase. Mol Cell Biol 1999;19:6379-95.

15. Chen X, Prywes R. Serum-induced expression of the CDC25A gene by relief of E2F-mediated repression. Mol Cell Biol 1999;19:4695-702.

16. Galaktionov K, Chen X, Beach D. CDC25A cell-cycle phosphatase as a target of c-myc. Nature 1996;382:511-7. 17. Barre B, Vigneron A, Coqueret $O$. The STAT3 transcription factor is a target for the Myc and retinoblastoma proteins on the $\mathrm{CDC} 25 \mathrm{~A}$ promoter J Biol Chem 2005;280:15673-81.

18. Busino L, Chiesa M, Draetta GF, Donzelli M. CDC25A phosphatase: combinatorial phosphorylation, ubiquitylation and proteolysis. Oncogene 2004;23:2050-6.

19. Sorensen CS, Syljuasen RG, Bartek J, Lukas J. ATR, Claspin and the Rad9-1-Hus1 complex regulate Chk1 and CDC25A in the absence of DNA damage. Cell Cycle 2004;3:e35-9.

20. Loffler H, Syljuasen RG, Bartkova J, Worm J, Bartek J, Lukas J. Distinct mode of deregulation of the protooncogenic CDC25A phosphatase in human breast cancer cell lines. Oncogene 2003;22:8063-71.
21. $\mathrm{Xu} \mathrm{O}$, Thompson JE, Carroll M. mTOR regulates cell survival after etoposide treatment in primary AML cells. Blood 2005;106:4261-8.

22. Brezak MC, Quaranta M, Contour-Galcera MO, et al Inhibition of human tumor cell growth in vivo by an orally bioavailable inhibitor of CDC25 phosphatases. Mol Cancer Ther 2005;4:1378-87.

23. Seminario MC, Precht P, Wersto RP, et al. PTEN expression in PTEN-null leukaemic T cell lines leads to reduced proliferation via slowed cell cycle progression. Oncogene 2003;22:8195-204.

24. Gao N, Flynn DN, Zhang Z, et al. $G_{1}$ cell cycle progression and the expression of $\mathrm{G}_{1}$ cyclins are regulated by $\mathrm{PI} 3 \mathrm{~K} / \mathrm{Akt} / \mathrm{mTOR} / \mathrm{p} 70 \mathrm{~S} 6 \mathrm{Kl}$ signaling in human ovarian cancer cells. Am J Physiol Cell Physio 2004;287:C281-91.

25. Iwata $S$, Ohashi $Y$, Kamiguchi $K$, Morimoto C. $\beta_{1}$ Integrin-mediated cell signaling in $\mathrm{T}$ lymphocytes. J Dermatol Sci 2000;23:75-86.

26. Puc J, Keniry M, Li HS, et al. Lack of PTEN sequesters Chk1 and initiates genetic instability. Cancer Cell 2005;7: 193-204.

27. Puc J, Parsons R. PTEN loss inhibits CHKl to cause double stranded-DNA breaks in cells. Cell Cycle 2005;4 e71-3.

28. Min YH, Eom JI, Cheong JW, et al. Constitutive phosphorylation of Akt/PKB protein in acute myeloid leukemia: its significance as a prognostic variable. Leukemia 2003;17:995-7.

29. Recher C, Beyne-Rauzy O, Demur C, et al. Antileukemic activity of rapamycin in acute myeloid leukemia. Blood 2005;105:2527-34.

30. Khaled A, Bulavin D, Kittipatarin C, et al. Cytokinedriven cell cycling is mediated through CDC25A. J Cel Biol 2005;169:755-63. 